\title{
Risk Factors of HIV Co-Infection and Sexual Behaviours in Patients with Gonococcal Infection in a STI's Clinic in Madrid
}

Ariza-Mejía MC ${ }^{1}$, García-García L¹, Puerta-López $\mathrm{T}^{2}$, Menéndez-Prieto $\mathrm{B}^{2}$, Vera-García $\mathrm{M}^{2}$, Clavo-Escribano $\mathrm{P}^{2}$, Ballesteros Martín $\mathrm{J}^{2}$, Rodriguez-Martín $\mathbf{C}^{2}$, Gil de Miguel $\mathbf{A}^{1}$, del Romero-Guerrero $\mathbf{J}^{2}$ and Gil-Prieto $\mathbf{R}^{\mathbf{1}^{*}}$

${ }^{1}$ Department of Preventive Medicine and Public Health and Medical Immunology and Microbiology, Rey Juan Carlos University, Madrid, Spain

${ }^{2}$ Sandoval Health Centre, Madrid Health Service, Madrid, Spain

\begin{abstract}
Introduction: During the past decade, the incidence of gonorrhoea has increased in Spain, mainly affecting the community of men who have sex with men. The objective of the present study was to describe the cases of gonococcal infection at a referral centre for sexually transmitted diseases in Madrid, as well as the factors associated with co-infection by the human immunodeficiency virus.
\end{abstract}

Methods: A retrospective cross-sectional study was performed of all of the cases of gonococcal infection that were diagnosed in 2010 at the Sandoval Health Centre of Madrid. Clinical histories, diagnostic tests, and sociodemographic and risk-behaviour questionnaires were analysed.

Results: Eighty-three per cent of the patients who were diagnosed with gonorrhoea were men who had engaged in sex with men, and $29 \%$ tested positive by serological screening for HIV. In the multiple logistic regression model the following factors were associated with the HIV co-infection: having a positive history of one or more sexually transmitted diseases (OR=57.44; $95 \% \mathrm{Cl}: 6.78-486.50)$, having a stable partner who was HIV-positive (OR=23.13; $95 \% \mathrm{Cl}: 2.44-219.36)$, having a concomitant syphilis diagnosis (OR=8.88; 95\% Cl: 1.90-41.37), having engaged in unprotected, insertive anal intercourse $(\mathrm{OR}=6.10 ; 95 \% \mathrm{Cl}: 2.52-14.76)$, and having engaged in high-risk sexual contacts while under the influence of alcohol or other drugs (OR=2.73; 95\% Cl: 1.13-6.62).

Conclusions: In the present study, a greater incidence of gonococcal infection and HIV co-infection was observed in men who had engaged in sex with men. Therefore, greater emphasis should be placed on the routine screening for sexually transmitted diseases and the targeting of education and health-promotion initiatives to highrisk communities.

Keywords: Human immunodeficiency virus; Neisseria gonorrhoeae; Sexually transmitted infection; Men who have sex with men

Abbreviations: CI: Confidence Interval; CT: Chlamydia trachomatis; EDO: Enfermedad de Declaración Obligatoria; HAART: Highly Active Antiretroviral Therapies; HAV: Hepatitis A Virus; HBV: Hepatitis B Virus; HIV: Human Immunodeficiency Virus; HPV: Human Papillomavirus; IQR: Interquartile Rate; MSM: Men who has sex with Men; OR: Odds Ratio; PCR: Polymerase Chain Reaction; RPR: The Rapid Plasma Regain; STIs: Sexually Transmitted Infections; TPPA: Treponema pallidum Particle Agglutinatio

\section{Introduction}

An increase in the incidence of gonococcal infections has been observed in Europe [1,2] during the past decade, primarily in the community of men who have sex with men (MSM) [3]. An increased incidence of gonococcal infections has also been confirmed in Spain, where the rate was 4.27 per 100,000 in 2010, exhibiting an increase of $202 \%$ from 2002 [4]. An outbreak of gonococcal infections was detected in Madrid among men who have sex with men /bisexual males in 2004 $[5,6]$.

Due to its short incubation time, gonorrhoea is a strong indicator of high-risk sexual behaviours, which could also facilitate the transmission of other sexually transmitted infections (STIs). Furthermore, the gonococcal infection increases the risk of acquiring and transmitting HIV $[7,8]$. In turn, a higher prevalence of gonorrhoea has been reported in patients infected with HIV [9]. In addition to the HIV infection, there are several other factors that influence the transmission of the gonococcal disease in developed countries. In Spain, these factors include alcohol and drug use, a large number of sexual partners, and being an immigrant from a region with a high incidence of gonococcal disease, such as Africa or Southeast Asia [10-12].

The Spanish system for Diseases of Mandatory Declaration (Enfermedades de Declaración Obligatoria - EDO) constitutes one of the basic pillars of epidemiological monitoring. This system provides information concerning the incidence of infection, but it does not describe the socio-demographic and epidemiological characteristics of the infected population [13].

The objective of the present study was to describe gonorrhoea patients and their sexual behaviours at the Sandoval Centre, referral centre for sexually transmitted infections in Madrid and to study the factors associated with HIV co-infection in gonorrhoea patients in order to contribute to improve the knowledge of the epidemiological

${ }^{*}$ Corresponding author: Ruth Gil Prieto, Preventive Medicine and Public Health \& Medical Immunology and Microbiology Department, Rey Juan Carlos University, Avda Atenas s/n, CP: 28922 Alcorcón (Madrid), Spain, Tel: +34914888646; Fax: +34914888955; E-mail: ruth.gil@urjc.es

Received July 16, 2013; Accepted August 29, 2013; Published September 05 2013

Citation: Ariza-Mejía MC, García-García L, Puerta-López T, Menéndez-Prieto B, Vera-García M, et al. (2013) Risk Factors of HIV Co-Infection and Sexual Behaviours in Patients with Gonococcal Infection in a STI's Clinic in Madrid. J AIDS Clin Res 4: 240. doi: 10.4172/2155-6113.1000240

Copyright: (C) 2013 Ariza-Mejia MC, et al. This is an open-access article distributed under the terms of the Creative Commons Attribution License, which permits unrestricted use, distribution, and reproduction in any medium, provided the original author and source are credited. 
situation and proposing adequate preventive measures that meet the needs of the population.

\section{Materials and Methods}

A retrospective descriptive study was performed, including all the cases of gonococcal infection (located in the urethra, cervix, pharynx, and anus) that were diagnosed in 2010 at the Sandoval Health Centre in Madrid.

The Sandoval Health Centre is specialized in the study and treatment of STIs and its main characteristic is the confidentiality of the patients. No personal identification is requested to be tested and treated in the Sandoval Health Centre. Any person suspected of having a STI can anonymously ask for medical assistance regardless their nationality, legal status in the country or their coverage in the Public Social Security System. Testing and treatment are free of charge for the patients. These particularities of the Sandoval Health Centre make of it a reference STI centre in the Region of Madrid that is very well known in some collectives in social exclusion, such as MSM, bisexuals, transsexuals, non-regularized immigrants or commercial sex-workers. More than 30000 patients are seen annually in the Centre; most of them are young adults that belong to all levels of socio-economic and educational status. Some patients are derived to Sandoval Health Centre from primary health centres in the Region of Madrid.

This investigation was conducted using socio-demographic and risk-evaluation information regarding STIs. This information was obtained using a specific questionnaire that collected information about high-risk sexual practices, comorbidities with other STIs, and harmful habits such as smoking, drug use and regular intake of alcohol. These questionnaires were filled in by nurses through interview during the first visit of the patient. No identification data (name, social security number) were collected. Data are included in a de-identified data base to ensure patient confidentiality. Only authorized professionals of the Sandoval Health Centre have access to the data.

All of the patients who attended the centre in 2010 and were diagnosed with gonococcal infection were included in the study. Patients ( $n=7)$ who attended the centre a second time in the same month after gonorrhea diagnosis and who displayed the same gonococcal infection, as they had at the previous consultation, were excluded from the analysis.

The microbiological diagnosis of the gonococcal infection was performed using gram staining and was subsequently confirmed by culturing on Thayer-Martin selective agar. All patients presenting urethral, rectal and/or cervical purulent discharge were tested for gonorrhoea and CT. Serologies to test for HIV, hepatitis and syphilis were also performed. Tests for other STIs, such as HPV, were performed only in suspicious cases.

In this study, the term "concurrent STI" refers to the gonococcal infection that is concomitant with HIV, syphilis, CT, and/or HPV. Various techniques were used to study the other concomitant STIs. A polymerase chain reaction (PCR) (Abbott, Diagnostics Division) was performed to detect infections by CT. Syphilis was diagnosed via the direct observation of Treponema pallidum under a dark-field microscope in samples from ulcers that were indicative of primary syphilis and via the following serologies: the rapid plasma reagin (RPR) test (Alere Healthcare), Treponema pallidum particle agglutination assay (TPPA) (MB Kit), and ELISA (IgG + IgM, Alere Healthcare). Concomitant HPV was diagnosed using the HPV DNA detection test with hybrid capture 2 (Digene ${ }^{ø}$ ). HIV antibodies were detected using the MEIA technique (AXSYM ${ }^{\triangleright}$ Abbott Diagnostics Division) and were confirmed by western blot analysis (BIO-RAD).

\section{Statistical analysis}

A descriptive analysis was performed of patients with a positive diagnosis of gonorrhoea. For analysis purposes MSM, bisexuals and one transsexual (male to female) were grouped. MSM/bisexual vs. heterosexual men was compared. Differences in counts of categorical responses by gender and sexual orientation as well as differences in HIV-comorbidity between independent groups were evaluated using the $\chi^{2}$ test. For the comparison of medians the Mann-Whitney $U$ test was performed. A backwards stepwise logistic regression model was developed to identify the factors that were independently associated with HIV co-infections in gonorrhoea positive patients. The statistical analyses were performed using the Statistical Package for Social Sciences (SPSS) for Windows, version 19.0 (Chicago, Illinois, USA). For all of these tests, values of $\mathrm{p}<0.05$ were considered to be statistically significant.

\section{Ethics}

This study was approved by the Rey Juan Carlos University Research Ethics Committee (Internal code 302012). This study complied in all respects with the ethical safeguards laid down by the Helsinki Declaration and its subsequent updates, and with Spanish legislation governing clinical research involving humans and protection of data of a personal nature (Data Protection Act: LO 15/1999 de 13 de Diciembre and RD 1720/2007 de 21 de Diciembre).

\section{Results}

There were a total of 30,193 STI consultations in the Sandoval Health Centre in 2010. Of those, 5,082 samples (16.8\%) were collected for the diagnosis of gonococcal infections. A total of 2,134 (42\%) of them corresponded to MSM/bisexuals patients. Of the total of MSM/ bisexual patients, $7 \%$ ( $n=152$ and $3 \%$ of the total of samples tested) were commercial sex workers. Twenty-three percent $(n=1,194)$ of the total tested samples corresponded to female commercial sex workers.

The gonococcus was isolated from $323(6 \%)$ of the 5,082 samples tested. Of those, 320 patients (response rate of $98.2 \%$ ) responded to the questionnaire. The majority $(92.5 \%, \mathrm{n}=299)$ of the patients who were diagnosed with gonorrhoea were men. Of those, 268 (83\% of the 323 positive cases) were MSM/bisexuals, and $32(10 \%)$ were heterosexual men. The remaining $7 \%(n=23)$ were heterosexual women and $1(0.3 \%)$ was a transsexual male to female. A subset of patients $(n=28 ; 8.7 \%)$ were commercial sex workers. Of them, $5 \%(n=16)$ were MSM/bisexuals, and $3.7 \%(\mathrm{n}=12)$ were women.

The median age of the patients diagnosed with gonorrhoea was 29 (IQR: 24-36) years, being significantly higher in MSM/bisexuals (33 years; IQR: $24-36)$ than in heterosexual men (28.6 years; IQR: 22-32.5) and women (27 years; IQR: $22-33$ ). The majority of the patients $(56.3 \%)$ were Spanish, 32.9\% came from Latin America and the Caribbean, 3.8\% were from Western Europe, 3.5\% were from Eastern Europe, $1.6 \%$ were from North America, another $1.6 \%$ were from Africa, and the remaining $0.6 \%$ were from Oceania. The majority $(46.5 \%)$ of the patients had higher education, $36.1 \%$ had secondary education, $16.8 \%$ had primary education, and only one patient $(0.3 \%)$ reported having no primary education.

The most common localization of the gonococcal infection was urethral (54.5\%), followed by rectal (32.2\%), oropharyngeal (5.9\%), and cervical (3.7\%). In men who have sex with men the main localizations 
were urethra (51.3\%), followed by rectum (37.1\%), oropharynge (6.2\%) and mixed locations (5.5\%). In heterosexual men the most common localizations of gonococcal infection was urethral $(73.6 \%)$, rectal $(20.7 \%)$, oropharyngeal $(3.8 \%)$ and mixed localizations $(1.9 \%)$, whereas in heterosexual women the most common localization was cervical (63.6\%), oropharyngeal $(18.2 \%)$ and cervical plus rectal (18.2\%). The majority (87.6\%) of the patients displayed clinical signs or symptoms, $90.5 \%$ from MSM, $96.2 \%$ from heterosexual men and $54.5 \%$ from heterosexual women. The most common manifestations of the urethral gonococcal infections were purulent urethral secretions (present in $100 \%$ of the cases) and dysuria (present in $12 \%$ of the cases). The most common symptoms of the rectal gonococcal infections were proctitis (present in $54.8 \%$ of the cases) and purulent rectal secretions (present in $19.1 \%$ of the cases). In men who have sex with men the most common symptomatology presented were purulent urethral secretions (53\%), proctitis $(23 \%)$ and purulent rectal secretions $(8.3 \%)$. In the heterosexual men the most common manifestations of gonococcal infection were purulent urethral secretions $(71.7 \%)$, proctitis $(11.3 \%)$, purulent rectal secretions $(5.6 \%)$, pharyngitis $(3.7 \%)$, pain in rectum (1.9\%) and pruritus urethral (1.9\%); whereas the most common manifestations in heterosexual women were purulent cervical secretions (27.3\%), cervicitis (18.2\%) and dysphagia (9.1\%).

Seventy per cent of the patients had had more than 50 partners during their lives, and more than $50 \%$ had had 10 or more partners within the past year; this latter figure was higher among the MSM/ bisexuals $(\mathrm{p}<0.001)$. The average age at the first sexual intercourse was 17 years, and it was higher for the MSM/bisexuals than for the heterosexuals ( $\mathrm{p}=0.003$ ) (Table 1). An important proportion (40.6\%) of the patients either currently or previously had a stable partner during the past year ( $4.1 \%$ of the MSM/bisexuals and $60.7 \%$ of the heterosexuals men); furthermore, $6.9 \%$ of the patients had a stable partner with a positive serology for HIV, and $90.4 \%$ kept a stable partner along with other, casual partners at the same time.

Of the 281 patients from whom information was obtained regarding high-risk sexual practices and harmful habits, 38.1\% reported being habitual users of recreational drugs, 38.4\% drank regularly alcohol, and $36 \%$ reported having sex while under the influence of drugs or alcohol both with stable partner and with casual partners.

Two thirds (67.2\%) of the patients used condoms both with stable partner and with sporadic contacts, and there were no significant differences between the MSM/bisexuals and heterosexuals. The frequency of several unprotected sexual behaviours between MSM/ bisexuals and heterosexual men is displayed in Table 1. A high percentage of high-risk sexual behaviours (unprotected sexual contacts) were observed both in MSM/bisexuals and in heterosexual men, the differences were not significant $(\mathrm{p}=0.16)$.

Sixty per cent $(n=194)$ of the patients who were diagnosed with gonorrhoea had a history of STIs (32\% had a previous case of gonorrhoea, $24.7 \%$ had syphilis, $24.7 \%$ had HPV, $17.3 \%$ had HIV, and $15.7 \%$ had CT). At the time of the consultation, the STIs that were concomitant with the gonococcal infection were predominantly CT (19.7\%), HPV (18.8\%), HIV (11.9\%), and syphilis (7.4\%). The HIV serology was positive for $29 \%(n=94)$ of the cases $(12 \%(n=39)$ were new diagnoses, and $17 \%(n=55)$ were already known). The patients with a positive HIV serology had had a higher number of partners within the past year $(\mathrm{p}<0.001)$. The MSM/bisexuals group comprised the majority of those patients who were infected with HIV (92.9\%); they also had a higher STI and HIV histories $(\mathrm{p}<0.001)$.

Thirty-six per cent $(n=116)$ of the patients exhibited a positive serology for the hepatitis virus; of these patients, $50.6 \%$ were positive for hepatitis A (HAV), 20.2\% for hepatitis B (HBV) (positive anti-HBc), $21 \%$ for HAV and HBV, and $2.2 \%$ for hepatitis C. The remaining $6 \%$ reported a history of hepatitis that was not specified in the clinical history. Almost two third (61.8\%) of the patients reported that they had been vaccinated against $\mathrm{HBV}$, and $32.8 \%$ reported being vaccinated against HAV. Vaccination coverage both for HAV and HVB was significantly higher ( 0.001 and $<0.001$, respectively) in MSM/bisexuals than in heterosexual men and heterosexual women.

The risk factors for the HIV co-infection in patients with gonorrhoea according to the bivariate analysis were the following: having a stable partner with a positive HIV serology, being MSM/bisexual, having a history of STIs, having a concomitant syphilis diagnosis, practicing unprotected insertive anal intercourse and/or receptive anal intercourse, having sexual contacts while under the influence of alcohol or drugs, and self-reported high consume of alcohol and drugs (Table 2). The prevalence of HIV co-infections was higher among the patients who practiced anal intercourse without a condom than among the patients who reported that they always used one (Table 2).

The multiple logistic regression model showed that the factors associated with co-infection with the human immunodeficiency virus in men diagnosed with gonorrhoea in the Sandoval Health Centre of

\begin{tabular}{|c|c|c|c|c|c|c|}
\hline Variable (statistic test used) & & Overall $(n=323)$ & MSM/Bisexuals $(n=268)$ & Heterosexuals men $(n=32)$ & Women $(n=23)$ & $p$ \\
\hline \multirow{4}{*}{$\begin{array}{l}\text { Frequency of unprotected sexual practices with a stable } \\
\text { partner }\end{array}$} & OGS $\left(x^{2}\right)$ & $93.9 \%$ & $97.9 \%$ & $21.8 \%$ & $81.8 \%$ & $<0.001$ \\
\hline & $\mathrm{VI}\left(\mathrm{X}^{2}\right)$ & $12.8 \%$ & $0.0 \%$ & $0.0 \%$ & $70 \%$ & NA \\
\hline & $\mid \mathrm{AI}\left(\mathrm{X}^{2}\right)$ & $44.5 \%$ & $71.6 \%$ & $3.1 \%$ & $0.0 \%$ & $<0.001$ \\
\hline & RAI $\left(X^{2}\right)$ & $39.6 \%$ & $56.6 \%$ & $0.0 \%$ & $33.3 \%$ & $<0.001$ \\
\hline \multirow{4}{*}{$\begin{array}{l}\text { Frequency of unprotected sexual practices with casual } \\
\text { partners }\end{array}$} & OGS $\left(x^{2}\right)$ & $88.4 \%$ & $96.2 \%$ & $56.25 \%$ & $75.1 \%$ & $<0.001$ \\
\hline & VI $\left(x^{2}\right)$ & $5.4 \%$ & $2.4 \%$ & $0.0 \%$ & $37.5 \%$ & $<0.001$ \\
\hline & $\mathrm{IAI}\left(\mathrm{X}^{2}\right)$ & $36.5 \%$ & $39.6 \%$ & $6.25 \%$ & $0.0 \%$ & $<0.001$ \\
\hline & RAI $\left(X^{2}\right)$ & $10 \%$ & $43.4 \%$ & $0.0 \%$ & $6.7 \%$ & $<0.001$ \\
\hline \multicolumn{2}{|c|}{ Median number of partners within the past year (IQR) (MWU test) } & $10(4.25-30.0)$ & $10(5-40.0)$ & $3.5(2.7-10)$ & $3.0(1.3-362.0)$ & $<0.001$ \\
\hline \multicolumn{2}{|l|}{ Median number of partners within the lifetime (IQR) (MWU test) } & $100(30-210)$ & $100(32.5-300)$ & $11.5(8.75-287.5)$ & $3.0(1.5-12.0)$ & $<0.001$ \\
\hline \multicolumn{2}{|c|}{ Frequency of unprotected sexual contacts during the past 3 months $(\%)\left(x^{2}\right)$} & $82.8 \%$ & $78.6 \%$ & $83.9 \%$ & $68 \%$ & 0.16 \\
\hline \multicolumn{2}{|l|}{ Median age at the first sexual activity (IQR) (MWU test) } & $17(15.0-18.0)$ & $17.0(16.0-18.0)$ & $16(14.0-18.0)$ & $15(14-18)$ & 0.003 \\
\hline \multicolumn{2}{|l|}{ Serology positive for HIV $\left(\mathrm{X}^{2}\right)$} & $29 \%$ & $33.5 \%$ & $3.2 \%$ & $4.3 \%$ & $<0.001$ \\
\hline
\end{tabular}

MSM: Men who have sex with men; OGS: orogenital sex; VI: vaginal intercourse; IAI: insertive anal intercourse; RAI: receptive anal intercourse; IQR: interquartile range MWU: Mann Whitney U.

Table 1: Characteristics of the sexual behaviours of patients diagnosed with gonorrhoea. 
Page 4 of 6

\begin{tabular}{|c|c|c|c|c|}
\hline \multicolumn{3}{|c|}{ Dependent variable: current HIV (co-infection with gonorrhoea) } & Crude OR $(95 \% \mathrm{Cl})$ & $\mathrm{p}$ \\
\hline \multirow{2}{*}{\multicolumn{2}{|c|}{ Gender }} & Man (300) & $4.76(1.11-20)$ & \multirow[t]{2}{*}{0.036} \\
\hline & & Woman (23) & - & \\
\hline \multirow{2}{*}{\multicolumn{2}{|c|}{ HIV-positive stable partner }} & Yes $(n=22)$ & $13.56(4.44-41.35)$ & \multirow{2}{*}{$<0.001$} \\
\hline & & No (301) & - & \\
\hline \multirow{2}{*}{\multicolumn{2}{|c|}{ Sexual orientation (men) }} & MSM/bisexual $(n=268)$ & $6.39(2.66-15.38)$ & \multirow{2}{*}{$<0.001$} \\
\hline & & Heterosexual men $(n=32)$ & & \\
\hline \multicolumn{3}{|l|}{ Age (years) } & $0.97(0,94-1)$ & 0.48 \\
\hline \multicolumn{3}{|c|}{ Age at first sexual intercourse (years) } & $0.87(0.78-0.96)$ & 0.11 \\
\hline \multirow{2}{*}{\multicolumn{2}{|c|}{ STD history }} & Yes $(n=193)$ & $56.93(13.68-236.99)$ & \multirow{2}{*}{$<0.001$} \\
\hline & & No $(n=130)$ & - & \\
\hline \multirow{2}{*}{\multicolumn{2}{|c|}{ Co-infection with Chlamydia trachomatis }} & Yes $(n=62)$ & $1.76(0.98-3.14))$ & \multirow{2}{*}{0.058} \\
\hline & & No $(n=257)$ & & \\
\hline \multirow{2}{*}{\multicolumn{2}{|c|}{ Co-infection with syphilis }} & Yes $(n=80)$ & $3.68(1.77-7.61)$ & \multirow{2}{*}{$<0.001$} \\
\hline & & No $(n=243)$ & - & \\
\hline \multirow{4}{*}{ Frequency of sexual behaviour } & \multirow{2}{*}{ IAI with SC } & Unprotected $(n=118)$ & $5.17(2.73-9.80)$ & \multirow{2}{*}{$<0.001$} \\
\hline & & Protected $(n=205)$ & - & \\
\hline & \multirow{2}{*}{ RAI with SC } & Unprotected $(n=32)$ & $4.46(2.39-8.31)$ & \multirow{2}{*}{$<0.001$} \\
\hline & & Protected $(n=291)$ & - & \\
\hline \multirow{2}{*}{\multicolumn{2}{|c|}{ Alcohol use }} & Yes $(n=124)$ & $2.35(1.40-3.96)$ & \multirow{2}{*}{0.010} \\
\hline & & No $(n=199)$ & - & \\
\hline \multirow{2}{*}{\multicolumn{2}{|c|}{ Non-injection drug use }} & Yes $(n=123)$ & $3.22(1.91-5.45)$ & \multirow{2}{*}{$<0.001$} \\
\hline & & No $(n=200)$ & - & \\
\hline \multirow{2}{*}{\multicolumn{2}{|c|}{$\begin{array}{l}\text { Sexual contacts under the influence of drugs or } \\
\text { alcohol }\end{array}$}} & Yes $(n=116)$ & $3.58(2.1-6.12)$ & \multirow{2}{*}{$<0.001$} \\
\hline & & No $(n=207)$ & - & \\
\hline
\end{tabular}

OGS: orogenital sex; IAI: insertive anal intercourse; RAl: receptive anal intercourse; SC: sporadic contact.

Table 2: Bivariate analysis. Factors associated with the human immunodeficiency virus co-infection in cases diagnosed with gonorrhoea. Sandoval Health Centre, Madrid, 2010.

\begin{tabular}{|c|c|c|}
\hline Dependent variable: current HIV (co-infection with gonorrhoea) & adjusted OR(95\% Cl) & \multicolumn{1}{|c|}{$\mathrm{p}$} \\
\hline Age & $0.99(0.93-1.06)$ & 0.973 \\
\hline STD history & $57.44(6.78-486.50)$ & $<0.001$ \\
\hline HIV-positive stable partner & $23.13(2.44-219.36)$ & 0.003 \\
\hline Concomitant syphilis & $8.88(1.90-41.37)$ & 0.005 \\
\hline Unprotected, insertive anal intercourse & $6.10(2.52-14.76)$ & $<0.001$ \\
\hline Sexual contacts under the influence of drugs/alcohol & $2.73(1.13-6.62)$ & 0.026 \\
\hline
\end{tabular}

The backward selection procedure removed the following variables from the model: Alcohol use, Non-injection drug use, Co-infection with Chlamydia trachomatis, Age at first sexual intercourse (years) and sexual orientation.

Table 3: Multiple logistic regression model of the factors associated with co-infection with the human immunodeficiency virus of men diagnosed with gonorrhoea. Sandoval Health Centre, Madrid, 2010.

Madrid in 2010 were as follows: having a history of STIs (OR=57.44; CI 95\% (6.78-486.50)); having an HIV-positive stable partner (OR=23.13; CI 95\% (2.44-219.36)); having a concomitant syphilis diagnosis $(\mathrm{OR}=8.88$; CI 95\% (1.90-41.37)); the practice of unprotected, insertive anal intercourse $(\mathrm{OR}=6.10$; CI $95 \%(2.52-14.76))$; and the practice of high-risk sexual contacts while under the influence of alcohol or drugs (OR=2.73; CI 95\% (1.13-6.62)) (Table 3).

\section{Discussion}

The number of gonococcal infections at the Sandoval Health Centre in 2010 ( 323 cases) was one of the highest in several years; for example, in 2005, only 134 cases of gonococcal infection were diagnosed [11]. This increase in the number of gonococcal infections is in line with the increase of gonococcal infections reported in Spain and in other European countries in the last years [14-16]. Various factors could have influenced this phenomenon, including 1) the impact of highly active antiretroviral therapies (HAART) on high-risk sexual behaviours, which has minimised the perception of STI transmission and associated morbidity burden and thereby reduced safe sexual behaviours [17], and 2) the possible increase of population of MSM [18].

Of the total patient population for whom gonorrhoea screenings were conducted at the centre, slightly more than 25\% were MSM. However, the majority of the new diagnoses (83\%) arose from this community. The proportion of the MSM among new gonococcal infections in this study was similar to the percentages that have been reported in other European publications $[15,18]$.

It is well known that inconsistent condom use combined with a larger number of sexual partners is associated with STI diagnoses in MSM [19]. Low condom use among MSM has been observed in our study, given that approximately $80 \%$ of them have had unprotected sexual contacts in the past three months; moreover, this group has the highest number of sexual partners. Approximately $40 \%$ have had unprotected anal intercourse (insertive or receptive) with casual partners, which suggests a higher risk of acquiring gonorrhoea and other STIs. In the present study, it was observed that patients who had a higher number 
of sexual partners used condoms more frequently. However, the protective effect of condom use disappears in our study when patients have multiple sexual partners. This unexpected effect can be explained by the inconsistent use of condoms, which leads to a risk that is similar to not using any prophylactic methods against the gonococcal infection and other STIs (such as HIV). In fact, upon comparing the frequency of condom use, it was observed that the prevalence of HIV infections was higher in those subjects who did not always use a condom during anal intercourse, compared with those who consistently used condoms.

Having a previous history of gonorrhoea increases the risk of reinfection [20]. In our study, the most frequent localizations of the gonococcal infection was urethral followed by rectal, oropharyngeal, and cervical; these findings may be influenced by the characteristics of our sample, patients presenting with active symptoms of infection, but are consistent with the results of an investigation conducted in eight western European countries for the detection of antibiotic resistance [21].

Furthermore, drug and/or alcohol use and sexual contacts under the influence of drugs and/or alcohol were independently associated in our sample with HIV and gonorrhoea co-infections, This relationship has also been reported in other studies in Spain, Great Britain, The Netherlands and Norway [22-25]. Other independent risk factors for HIV co-infections in our study were the presence of other STIs (such as syphilis) and having an HIV-positive stable partner, findings that are in line with those from studies in Western Europe [22]. Other authors have indicated that rectal gonorrhoea is an independent risk factor for HIV transmission [9].

The recent increase in new diagnoses of sexually transmitted HIV infections compared with the decrease in transmission by the use of injected drugs [26] along with the low frequency of condom use, highlights the persistence of high-risk sexual behaviours in the general Spanish population, as well as in other countries such as Australia [2729]. Our study supports this observation in a particular subset of the Spanish population living in the capital of Spain.

One of the main limitations of this study was the use of selective cultures for diagnosing gonorrhoea. These cultures have a low sensitivity for anal and pharyngeal samples; consequently, the number of diagnoses could be underestimated. It should be noted that the data were obtained from voluntary screening tests that were performed at a health centre specialising in STI/HIV; thus, these data were subject to selection bias. Asymptomatic infections may have been underreported in our study. The present study cannot provide information about the prevalence of gonorrhoea/HIV co-infections among the rest of the population in Spain. However, our results in a population that is usually underrepresented in health research suggest that certain highrisk sexual behaviours (such as unprotected anal intercourse) lead to co-infections by HIV and the gonococcus. They also allow us to describe some characteristics of the patients that are affected by these co-infections such as previous STI history the HIV status of the partner. This type of information cannot be obtained through the current epidemiological monitoring systems.

The width of the confidence intervals from the logistic regression analysis indicates that the single effect estimates are imprecisely estimated. This is a direct consequence of the small sample size.

A review of STI prevention measures is necessary in addition to the implementation and improvement of sex-education campaigns that are especially directed to the MSM community. This proposal is similar to those measures that are currently conducted with notably positive results in other countries, such as the US [30]. These campaigns promote appropriate sex education (beginning in childhood) and condom use as a barrier method to STIs, and their messages provide warnings of the risk of engaging in sexual activity while under the influence of alcohol and drugs.

Furthermore, the creation of a greater number of STI clinics that are available for all citizens providing easy and widespread access without administrative barriers is crucial because these clinics are strategic health-assistance facilities that permit the early diagnosis of STIs, thereby breaking the transmission chain of infection and facilitating the early diagnosis and treatment of STIs, as well as tracing of contacts.

\section{Conclusions}

- A total of $83 \%$ of those patients who were diagnosed with gonorrhoea in 2010 at the Sandoval Health Centre were MSM.

- It is necessary to implement sex-education campaigns that are especially directed to the MSM community.

\section{Acknowledgments}

The authors thank the entire Sandoval Health Centre and, in particular, nurses Natividad Jerez, $\mathrm{M}^{\mathrm{a}}$ Ángeles Neila, and Sonsoles del Corral for their excellent healthcare and assistance in the data collection.

\section{References}

1. Fenton KA, Lowndes CM (2004) Recent trends in the epidemiology of sexually transmitted infections in the European Union. Sex Transm Infect 80: 255-263.

2. European centre for Disease Prevention and Control (2011) Sexually Transmitted infections in Europe 1990-2009. Stockholm, ECDC.

3. Johansen JD, Smith E (2002) Gonorrhoea in Denmark: high incidence among HIV-infected men who have sex with men. Acta Derm Venereol 82: 365-368.

4. Situación de las Enfermedades de Declaración Obligatoria (2010) Madrid: Instituto de Salud Carlos III Institute of Health.

5. Menéndez B, Ballesteros J, Clavo P, del Romero J (2005) [Increase in syphilis and gonorrhoea among men who have sex with men in Madrid]. Med Clin (Barc) 125: 756.

6. Ariza-Mejía MC, García-García L, Alvaro-Meca A, Gil-de-Miguel A, Gil-Prieto R (2010) [Gonococcal infection related hospital admissions in Madrid: 19972006]. Enferm Clin 20: 222-228.

7. Zhang J, Li G, Bafica A, Pantelic M, Zhang P, et al. (2005) Neisseria gonorrhoeae enhances infection of dendritic cells by HIV type 1. J Immunol 174: 7995-8002.

8. Rius C, Binefa G, Casabona J (2004) [Epidemiology of HIVIAIDS infection and relationship with other sexually transmitted diseases (STD). Future prospects] Enferm Infecc Microbiol Clin 22: 419-429.

9. Cohen MS (2004) HIV and sexually transmitted diseases: lethal synergy. Top HIV Med 12: 104-107.

10. Corbeto EL, Lugo R, Martró E, Falguera G, Ros R, et al. (2011) [Prevalence and determining factors of acquiring $C$. trachomatis infection among adolescents and young adults in Catalonia]. Enferm Infecc Microbiol Clin 29: 96-101.

11. Cano S, Fuentes M, Ballesteros J, Clavo P, Menéndez B, et al. (2009) [Gonorrhea diagnoses in a center for sexually transmitted disease (STD) and their relationship with HIV and other STD, Madrid, 2005]. Enferm Infecc Microbiol Clin 27: 338-341.

12. Postigo C (2007) [Sexually transmitted diseases and immigration in Spain]. Actas Dermosifiliogr 98: 513-517.

13. Díaz-Franco A, Noguer-Zambrano I, Cano-Portero R (2005) [Epidemiologica surveillance of sexually-transmitted diseases. Spain 1995-2003]. Med Clin (Barc) 125: 529-530

14. Macdonald N, Dougan S, McGarrigle CA, Baster K, Rice BD, et al. (2004) Recent trends in diagnoses of HIV and other sexually transmitted infections in England and Wales among men who have sex with men. Sex Transm Infect 80: 492-497. 
Citation: Ariza-Mejía MC, García-García L, Puerta-López T, Menéndez-Prieto B, Vera-García M, et al. (2013) Risk Factors of HIV Co-Infection and Sexual Behaviours in Patients with Gonococcal Infection in a STI's Clinic in Madrid. J AIDS Clin Res 4: 240. doi: 10.4172/2155-6113.1000240

Page 6 of 6

15. Velicko I, Unemo M (2009) Increase in reported gonorrhoea cases in Sweden, 2001 - 2008. Euro Surveill 14

16. Velicko I, Unemo M (2012) Recent trends in gonorrhoea and syphilis epidemiology in Sweden: 2007 to 2011. Euro Surveill 17.

17. Murray JM, Prestage G, Grierson J, Middleton M, McDonald A (2011) Increasing HIV diagnoses in Australia among men who have sex with men correlated with the growing number not taking antiretroviral therapy. Sex Health 8: 304-310.

18. Lepe Jiménez JA, Otero Guerra L, Blanco Galán MA, Aznar Martín J, Vázquez Valdés $F$ (2008) [Update on the epidemiology, diagnosis and treatment of sexually-transmitted infections]. Enferm Infecc Microbiol Clin 26 Suppl 13: 2531.

19. Martin IM, Hoffmann S, Ison CA; ESSTI Network (2006) European Surveillance of Sexually Transmitted Infections (ESSTI): the first combined antimicrobial susceptibility data for Neisseria gonorrhoeae in Western Europe. J Antimicrob Chemother 58: 587-593.

20. Fowler T, Caley M, Johal R, Brown R, Ross JD (2010) Previous history of gonococcal infection as a risk factor in patients presenting with gonorrhoea. Int J STD AIDS 21: 277-278.

21. Hughes G, Nichols T, Ison CA (2011) Estimating the prevalence of gonococcal resistance to antimicrobials in England and Wales. Sex Transm Infect 87: 526531.

22. Scoular A, Abu-Rajab K, Winter A, Connell J, Hart G (2008) The case for social marketing in gonorhoea prevention: insights from sexual lifestyles in Glasgow genitourinary medicine clinic attendees. Int J STD AIDS 19: 545-549.

23. Jakopanec I, Schimmer B, Grjibovski AM, Klouman E, Aavitsland P (2010) Self- reported sexually transmitted infections and their correlates among men who have sex with men in Norway: an Internet-based cross-sectional survey. BMC Infect Dis 10: 261.

24. Young H, McElhinney J, Palmer HM; Scottish gonococcal surveillance contributors (2006) Extended surveillance of gonorrhoea in Scotland 2003. Int J STD AIDS 17: 687-692.

25. Heiligenberg M, Wermeling PR, van Rooijen MS, Urbanus AT, Speksnijder AG, et al. (2012) Recreational drug use during sex and sexually transmitted infections among clients of a city sexually transmitted infections clinic in Amsterdam, the Netherlands. Sex Transm Dis 39: 518-527.

26. Moreno C, Irisarri F, Urtiaga M, Sola J, Fernández M, et al. (2003) [Epidemiological surveillance of HIV/Aids infections in Navarra between 1985 and 2002]. An Sist Sanit Navar 26: 269-275

27. Encuesta de Salud y Hábitos sexuales (2004) Salud y hábitos sexuales. Madrid: Instituto Nacional de Estadística y Ministerio de Sanidad y Consumo.

28. Ryder N, Bourne C, Donovan B (2011) Different trends for different sexually transmissible infections despite increased testing of men who have sex with men. Int J STD AIDS 22: 335-337.

29. Vodstrcil LA, Fairley CK, Fehler G, Leslie D, Walker J, et al. (2011) Trends in chlamydia and gonorrhea positivity among heterosexual men and men who have sex with men attending a large urban sexual health service in Australia, 2002-2009. BMC Infect Dis 11: 158

30. Johnson WD, Diaz RM, Flanders WD, Goodman M, Hill AN, et al. (2008) Behavioral interventions to reduce risk for sexual transmission of HIV among men who have sex with men. Cochrane Database Syst Rev: CD001230. 